\title{
Nosing around for new antibiotics
}

\section{ce}

Lugdunin

had potent

antimicrobial

activity against

Gram-positive bacteria

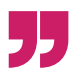

New antibiotics are urgently needed to address the growing problem of drug resistance. Now, Peschel and colleagues have identified an antibacterial compound - lugdunin - produced by a nasal commensal bacteria. Importantly, lugdunin has a novel chemical structure, which could provide a basis for a new class of antibiotics.

Most currently used antibiotics are derivatives of compounds isolated from soil-dwelling actinomycetes in the 1940s to 1960s. In this article, the authors screened a collection of nasal staphylococcal isolates

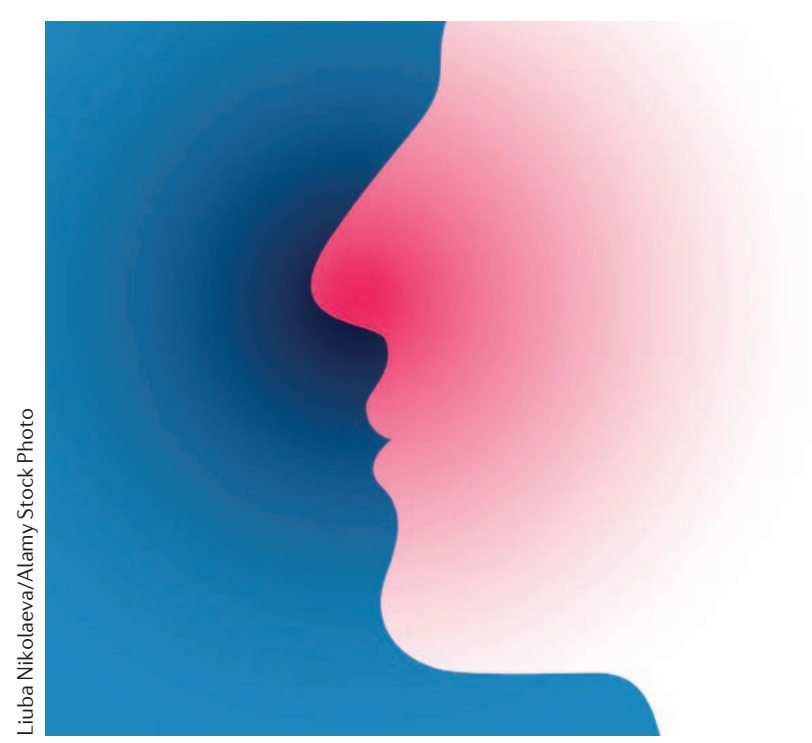

for activity against Staphylococcus aureus, a common human pathogen, and found a strain of Staphylococcus lugdunensis that strongly suppressed the growth of S. aureus. An operon in S. lugdunensis was subsequently identified that contains several genes related to antibiotic biosynthesis, all with less than 35\% identity to any other described enzyme, and an uncharacterized gene encoding a putative non-ribosomal peptide synthase. These data suggested that S. lugdunensis could produce a novel, non-ribosomally synthesized peptide antibiotic.

The authors then isolated and characterized lugdunin, a cyclic peptide with an unusual thiazolidine heterocycle and five amino acids. Lugdunin had potent antimicrobial activity against Gram-positive bacteria, including vancomycin-resistant Enterococcus spp. and S. aureus strains with resistance to methicillin or intermediate resistance to glycopeptide antibiotics. This compound seems to specifically kill bacteria, as it did not lyse primary human neutrophils or erythrocytes. Resistance was not observed under continued exposure to subinhibitory concentrations for 30 days.

In a mouse model of $S$. aureus skin infection, topical treatment with a lugdunin ointment 24, 30 and
42 hours after infection strongly reduced or eradicated viable $S$. aureus on the surface and in the deep layers of the skin. Lugdunin resistance was not found in any of the remaining viable $S$. aureus isolates.

Lugdunin-producing S. lugdunensis strains outcompete $S$. aureus in the noses of cotton rats. Furthermore, S. aureus and S. lugdunensis were found in $32.1 \%$ and $9.1 \%$ of nasal swabs from hospitalized patients, respectively, but these two species were not commonly found in the same swab: S. lugdunensis-colonized patients had a 5.9\%-fold lower tendency to carry S. aureus than S. lugdunensis-negative patients did.

Lugdunin or derivatives thereof could become therapeutically useful for the treatment of Gram-positive infections. Alternatively, lugduninproducing commensal strains of staphylococci might be used prophylactically in high-risk patients. Furthermore, the discovery of lugdunin indicates that human-associated microbes could be an untapped source for antibiotic discovery.

Megan Cully

ORIGINAL ARTICLE Zipperer, A. et al. Human commensals producing a novel antibiotic impair pathogen colonization. Nature 535, 511-516 (2016) FURTHER READING Tomassi, R. et al. ESKAPEing the labyrinth of antibacterial discovery. Nat. Rev. Drug Discov. 14, 529-542 (2015) 